\title{
OPERATIONS ON CONVERSATIONAL MIND-GRAPHS
}

\author{
Jayanta Poray, Christoph Schommer \\ Department of Computer Science and Communication, University of Luxembourg, 6, Rue Coudenhove-Kalergi, L-1359, \\ Luxembourg \\ \{jayanta.poray, christoph.schommer\}@uni.lu
}

\begin{abstract}
Keywords: Adaptive Information Management, Learning, Associative Memories, Linguistic Processing, Graph Operations.

Abstract: $\quad$ Mind-graphs define an associative-adaptive concept of managing information streams, like for example words within a conversation. Being composed of vertices (or cells; representing external stimuli like words) and undirected edges (or connections), mind-graphs adaptively reflect the strength of simultaneously occurring stimuli and allow a self-regulation through the interplay of an artificial 'fever' and 'coldness' (capacity problem). With respect to this, an interesting application scenario is the merge of information streams that derive from a conversation of $\mathrm{k}$ conversing partners. In such a case, each conversational partner has an own knowledge and a knowledge that (s)he shares with another. Merging the own (inside) and the other (outside) knowledge leads to a situation, where things like e.g. trust can be decided. In this paper, we extend this concept by proposing extended mind-map operations, dealing with the merge of sub-mind-graphs and the extraction of mind-graph skeletons.
\end{abstract}

\section{INTRODUCTION}

Today, the exchange of textual information by electronic devices is very popular. It ranges from simple short-term messages to collections of conversations, which have obtained by several months or years. And in fact, the produce of textual information within a conversation is a non-deterministic process, which requires a linguistic preparation of the texts, and a computational finesse, if the generated information is to be accumulated or summarised with regards to content. As one of the most promising research topic in the next years, the exploration of chats inside social networks belongs to this category [Tuulos and Tirri, 2004] .

Some research work has been done in the field of information accumulation, but the handling of a dynamic conversation within an adaptive framework has mostly been solved by associative graphs and the representation of information within these graphs. For example, a text summarisation method LexRank has been suggested by [Radev, 2004], where each vertex corresponds to the extracted topic from the input text and connection to the relation between several topics. In [Poray and Schommer, 2009] it has been shown that each conversing person can receive an understanding of its partner, if all incoming textual stimuli are linguistically processed and then put to a associative framework (mind-graph): the idea is that strong and weak connections - which emerge depending on the intensity and frequency of the signals - then finally lead to even such associative mindgraphs, which do not only reflect a textual conversation but moreover support a mental representation of the "other". And of course, a mental representation inside an associative framework can also be performed to the self of a person: a similarity (and dissimilarity) of these mind-graphs (the other's and the self) can become highly interesting regarding a mutual estimation (confidence, trust, et cetera).

As per the general continuation of this approach, a refinement concerns the categorisation of the information - which occurs during a conversation - into several categories, which we call a) known, b) $\mathrm{mu}$ tual, and c) unknown information (Figure 1). Category a) refers to information that is already aware by a person before a conversation takes place and that is already inside the associative mind-graph; b) refers to a common information between several conversing partners: it evolves over time and is then sent to the associative framework. Finally, c) Unknown information refers to information, which is not aware by a person before the conversation.

In this context, it is fair to confront the mind-graph framework to the Extended Mind Theory, which has been presented by (among others, but mainly) 
[Clark and Chalmers, 1998]. Here, it is suggested that external entities should be handled separately in the form of an active externalism and that mostly the internal and external information can not be separated, but considered as a coupled system. Also, an embodiment treats cognition as a set of tools evolved by organisms for coping with their environments ([Anderson, 2003]). It can be described as a control system of the body to aid a survival and reproductive success. Therefore, each entity not represents only with its sole existence, but mostly consider as the adapted form with its environment.
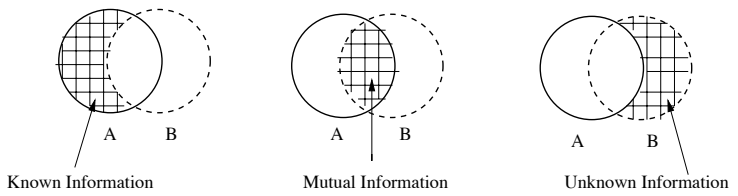

Figure 1: Three types of information during textual conversation (explanation see in text).

\section{Related Work}

The role of machine learning for automated text classification (or categorization) is discussed (among others) in [Sebastiani, 2002] and [Ikonomakis et al., 2005]. They clearly point out that an intersection of research fields like information extraction, text retrieval, summarization, question-answering et cetera exists particularly then, when the inductive process of learning has been motivated by the texts. With respect to the set-up of the relation among social communities, [Ziegler and Golbeck, 2006] show how today's online communities allow their users to find the co-relation to measure trust and interests. An enormous number of efforts has been made to use graph structures for a representation model [Cook and Holder, 2000]. In the work by [Yang, 1994] an expert network (ExpNet) has been proposed for automatic categorization and retrieval of natural language texts, where a word category ranking has been supported by the ExpNet framework.

In a similar approach, [Haghighi et al., 2005] has developed a graph matching model for sentence inference from texts. Many related approaches regarding the graph representation for texts and documents have been proposed since last few years by [Mani, 1997], [Montes-y Gómez et al., 2000], [Schenker et al., 2003] and [Hensman, 2004] . Recently, [Jin and Srihari, 2007] used a novel graph based text representation model capable to capture $a$ ) term order b) term-frequency c) term co-occurrance, and $d$ ) term context in a document; then test has been performed for a specific text mining task. The state-of-the-art of our proposed graph similarity based text representation model is mostly motivated by these research efforts.

\section{Operations on Mind-Graph}

With respect to the life-circle process of a textual information, raw conversational text data is treated firstly as the linguistic pre-processer. This includes a tokenization, the elimination of stop-words, a resolution of pronouns, and others. A temporary storage space, termed as Short Term Memory (STM), which contains this filtered information, is then used. For each set of conversational text (document), information is represented as an undirected graph ([Jin and Srihari, 2007]), called mind-graphs $(g=\{V, E\})$, which on their way represent a preprocessed conversational text by a set of vertices $(V)$ and a set of weighted edges $(E)$.

\subsection{Inside and Outside}

A mind-graph assimilates textual components and assigns each component to a vertex. Components that occur together are consequently bidirectionally connected and logistically managed inside a STM (Short Term Memory). It is connected to the LTM (Long Term Memory), which administers those mind-graphs that have proven a stability over time ([Poray and Schommer, 2010]). Concerning the "self" (Inside) and the "other" (Outside) as mentioned in section 1, Figure 2 reflects this situation, where each conversational partner keeps an own information as Inside and newly obtained information as Outside.

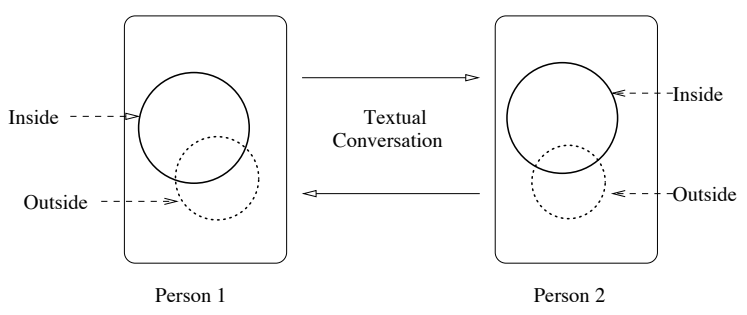

Figure 2: During textual conversation each person store their information as Inside and Outside

\subsection{Merge}

In a conversation between two or more individuals, the merge of the (prepared) stimuli is one of the essential task and consequence. Here, the grade of similarity between more than one type of information (see 
Section 1) has to be considered. In an extended situation of Figure 1, a merge (function) actually represents the similarity (intersection) between two types of information.

Definition 1. A merge function $\mu$ for two mindgraphs $g \in G$ and $g^{\prime} \in G$ (where $G$ is the set of mindgraphs) is defined as a one-to-one mapping among them, estimating the maximum common (similar) attributes among two mind-graphs.

$$
\mu\left(g, g^{\prime}\right): g \rightarrow g^{\prime}
$$

This similarity measurement using merge function reflects the amount of mutual information between Inside and Outside.

A mind-graph $g=(V, E, \lambda, \Delta)$ consists of a set of vertices $(V)$ and a set of edges $(E)$. Here, $\lambda: V \rightarrow L_{V}$ represents the identifier of a vertex, such that $\lambda(m) \neq$ $\lambda(n), \forall m, n \in V, m \neq n$ and $\Delta: E \rightarrow \mathbb{R}^{+}$(where $\Delta$ is the number of traffic observed in a labeled graph). Each mind-graph can be considered as the assemble of the different sub-graphs. Therefore, for $n$ different mindgraphs $g^{1}, \ldots, g^{n}$, a mutual similarity is given by their maximum common sub-mind-graph $\left(\operatorname{mcs}\left(g^{1}, \ldots, g^{n}\right)\right.$. Following [Bunke and Shearer, 1998], the distance (d) between these two mind-graphs is then defined as follows:

$$
d\left(g^{1}, \ldots, g^{n}\right)=1-\frac{\left|\operatorname{mcs}\left(g^{1}, \ldots, g^{n}\right)\right|}{\max \left\{\left|g^{1}\right|, \ldots,\left|g^{n}\right|\right\}}
$$

As an example (see Figure 3), for two mindgraphs $g$ and $g^{\prime}$ having the number of vertices $|g|,\left|g^{\prime}\right|$ respectively, also $\left|m c s\left(g, g^{\prime}\right)\right|$ represent the number of vertices for their maximum common sub-mind-graph $m c s\left(g, g^{\prime}\right)$. Since $|g|=6,\left|g^{\prime}\right|=4$ and $\left|m c s\left(g, g^{\prime}\right)\right|=3$, $d\left(g, g^{\prime}\right)=0.5$. Having the similarity as the complement of the the distance $d$ between $g$ and $g^{\prime}$, the formula is then

$$
\mu\left(g, g^{\prime}\right)=1-d\left(g, g^{\prime}\right)
$$

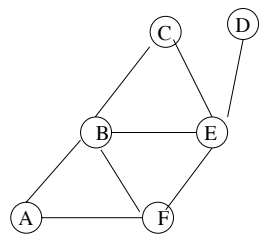

a)

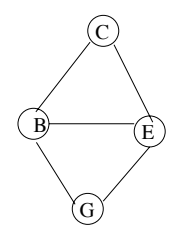

b)

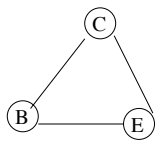

Figure 3: mind-graphs a) $g$ and; b) $g^{\prime}$; and their c) maximal common sub-graph, $\operatorname{mcs}\left(g, g^{\prime}\right)$

\subsection{Bags of Information}

As stated, the merge function $\mu$ is a real valued function, which represents the similarity between vertices (cells) and the associated connections of the mind-graphs [Poray and Schommer, 2009]. Actually, the degree of similarity corresponds to the common information inside these mind-graphs. Moreover, the common information with different merged value is being separated into different "bags", where each of this bag contains similar type of information. Let us consider that, there are $n$ number of topics (i.e., vertex of the mind-graph) obtained and these need to be placed into $k$ number of bags. Normally, $k<<n$, since a bag should contains more than one topic. Then the coding could be done as per the following rule:

if $\min _{i}<\mu\left(g, g^{\prime}\right) \geq \max _{i}$ then $b_{i}=+1$ else $b_{i}=-1$, where $i \in\{1, \ldots, k\}, \max _{i}=\min _{i+1}$ and $b_{i}$ is the $i^{t h}$ bag.

Here we simply categorize the similar values as per its grade. This also reflect the common interest about some specific topic. Threshold values are corresponds to the maximum $\left(\max _{i}\right)$ and minimum $\left(\min _{i}\right)$ values. These are obtained by the training data. In this process first the identify similar information and then separates corresponding vertices (cells) into different bags.

\subsection{Extraction of Skeletons}

As described in [Poray and Schommer, 2009], a skeleton is a mind-graph with strong connections (threshold) between its vertices. The extraction procedure follows an algorithm, which is described in the following. It follows two steps. In a first step, the graph potential for the weighted mind-graph is computed whereas in the second step the actual extraction is done:

(Step1)

Require: The set of weighted nodes $\left\{\omega_{1}, \ldots, \omega_{m}\right\}$

Require: The set of weighted connections $\left\{\omega_{i j}\right\}, i, j \in\{1, \ldots, m\} \& i \neq j$

$\alpha_{p} \leftarrow \frac{1}{m} \sum_{i=1}^{m} \omega_{i}$

$\beta_{p} \leftarrow \frac{1}{n} \sum_{k=1}^{n} \omega_{i j}$, where, $n=\left|\omega_{i j}\right|, i, j \in\{1, \ldots, m\}$ and $i \neq j$

Compute the graph potential $: \delta_{p} \leftarrow \frac{\alpha_{p}+\beta_{p}}{2}$

and (Step 2)

Require: The skeleton threshold $\delta_{s}$ if $\delta_{p} \geq \delta_{s}$ then 


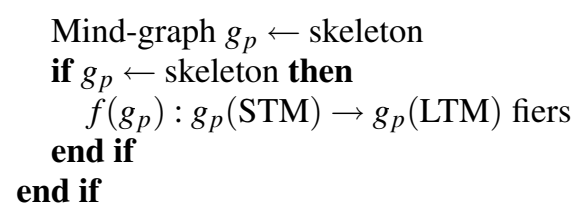

First, $\alpha_{p}$, which denotes the average value of all weighted nodes $\omega_{i}$ and $\beta_{p}$, which denotes the average value of all weighted connections $\omega_{i j}$, are considered. Thereafter, the graph potential $\delta_{p}$, which is the overall weighted average of that graph is obtained. Then (second step), the graph potential $\delta_{p}$ is compared with some pre-defined skeleton threshold $\delta_{s}$ and finally as per its grade, the skeletons are identified.

\subsection{Mind-graph normalization}

Sometimes, the mind-graphs need to be managed properly, such that the complexity of the graph always keep below a certain threshold value and maintain its healthy status. To keep the mind-graph in a consistent (normalized) state, it is advisable to consider only the connections, which do not a threshold value. The process of decomposition, join and selection is motivated to resolve this issue.

As an example in figure 4 the mind-graph $G 1$ is estimated as the "over graph threshold value" with too many complex connections. First this is decomposed into five subgraphs $A, B, C, D$ and $E$. Suppose among these five sub-graps $B$ and $C$ are again pointed as "over graph threshold value". Therefore, only rest three sub-graphs are taken for join. Similarly, in the selection phase the mind-graphs $A D$ and $D E$ are considered. Again the mind-graph $A E$ not selected as it is in "over graph threshold value" state.

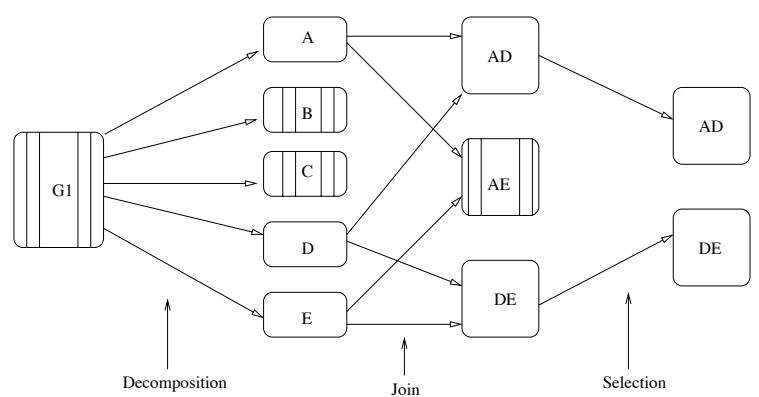

Figure 4: The data flow for Decomposition, Join and Selection for the mind-graph

The algorithm for this described technique is presented below, where only the candidate mind-graphs $g_{r}$ with "below graph threshold value" are stored inside the graph storage stack $\tilde{G}$.

Require: The mind-graph $g_{p}$ with its set of weighted nodes $\tilde{N}=\left\{\omega_{1}, \ldots, \omega_{m}\right\}$ and their weighted connections $\tilde{C}$.
Ensure: $\tilde{G} \leftarrow \emptyset$

1: Compute the graph potential $\delta_{p}$ of the input graph $g_{p}$

2: for $r=2$ to $m$ do

3: $\quad \tilde{N} \leftarrow\left\{\omega_{i}\right\}$, where $|\tilde{N}|=r$ and $i \in\{1, \ldots, m\}$

4: $\quad \tilde{C} \leftarrow\left\{\omega_{i j}\right\}$, where $i, j \in\{1, \ldots, m\}(i \neq j)$

5: $\quad \delta_{r} \leftarrow$ Compute graph potential for all subgraphs formed with $\tilde{N}$ and $\tilde{C}$

6: $\quad$ if $\delta_{r} \geq \delta_{p}$ then

7: DECOMPOSITION

8: JOIN

9: $\quad$ Get the candidate graph $g_{r}$

10: $\quad \tilde{G} \leftarrow g_{r}$

11: end if

12: end for

13: SELECTION (of the candidate graph(s) $\left(\leq \delta_{p}\right)$ from $\tilde{G}$ )

These two techniques are motivated to extract the skeleton mind-graph and manage the mind-graph complexity for complex connections. Also there exists some other techniques or can be formalized these as per some other specific need of the mind-graphs.

\section{Conclusions}

In this paper we used graphs (mind-graphs) to represent the coupling between the knowledge in the course of textual conversation. The similarity measures between the mind-graphs have been considered for information representation. Also, the algorithms associated to the mind-graphs extraction and normalization have been formalized. Initial experimental results are already achieved. It works with test sentences, where each word cells and their associated neighbor cells inside the mind-graph explicitly defined. Currently, we continue the test with a larger corpus. Other experimental observations associate with the module for mind-graph monitoring, extraction of other associated rules are also in consideration.

\section{Acknowledgement}

The current work has been performed at the University of Luxembourg within the project EAMM. We thank all project members for their support and engagement. 


\section{REFERENCES}

[Anderson, 2003] Anderson, M. L. (2003). Embodied cognition: A field guide. Artificial Intelligence, 149:91-130.

[Bunke and Shearer, 1998] Bunke, H. and Shearer, K. (1998). A graph distance metric based on the maximal common subgraph. Pattern Recogn. Lett., 19:255-259.

[Clark and Chalmers, 1998] Clark, A. and Chalmers, D. (1998). The extended mind. In Analysis, volume 58, pages 7-19.

[Cook and Holder, 2000] Cook, D. J. and Holder, L. B. (2000). Graph-based data mining. IEEE Intelligent Systems, 15:32-41.

[Haghighi et al., 2005] Haghighi, A. D., Ng, A. Y., and Manning, C. D. (2005). Robust textual inference via graph matching. In Proceedings of the conference on Human Language Technology and Empirical Methods in Natural Language Processing, HLT '05, pages 387-394. Association for Computational Linguistics.

[Hensman, 2004] Hensman, S. (2004). Construction of conceptual graph representation of texts. In Proceedings of the Student Research Workshop at HLT-NAACL 2004, HLT-SRWS '04, pages 49-54. Association for Computational Linguistics.

[Ikonomakis et al., 2005] Ikonomakis, M., Kotsiantis, S., and Tampakas, V. (2005). Text classification: a recent overview. In Proceedings of the 9th WSEAS International Conference on Computers.

[Jin and Srihari, 2007] Jin, W. and Srihari, R. K. (2007). Graph-based text representation and knowledge discovery. In Proceedings of the 2007 ACM symposium on Applied computing, SAC '07, pages 807-811. ACM.

[Mani, 1997] Mani, I. (1997). Multi-document summarization by graph search and matching. In In Proceedings of the Fifteenth National Conference on Artificial Intelligence (AAAI-97, pages 622-628. AAAI.

[Montes-y Gómez et al., 2000] Montes-y Gómez, M., López-López, A., and Gelbukh, A. F. (2000). Information retrieval with conceptual graph matching. In Proceedings of the 11th International Conference on Database and Expert Systems Applications, DEXA '00, pages 312-321. Springer-Verlag.

[Poray and Schommer, 2009] Poray, J. and Schommer, C. (2009). A cognitive mind-map framework to foster trust. In Proceedings of the 2009 Fifth International Conference on Natural Computation - Volume 05, ICNC '09. IEEE Computer Society.

[Poray and Schommer, 2010] Poray, J. and Schommer, C. (2010). Managing conversational streams by explorative mind-maps. In Proceedings of the ACS/IEEE International Conference on Computer Systems and Applications - AICCSA 2010. IEEE Computer Society.

[Radev, 2004] Radev, D. R. (2004). Lexrank: Graph-based lexical centrality as salience in text summarization. Journal of Artificial Intelligence Research, 22.

[Schenker et al., 2003] Schenker, A., Last, M., Bunke, H., and Kandel, A. (2003). Classification of web documents using a graph model. In Seventh International Conference on Document Analysis and Recognition, pages 240-244.

[Sebastiani, 2002] Sebastiani, F. (2002). Machine learning in automated text categorization. ACM Comput. Surv., $34: 1-47$.

[Tuulos and Tirri, 2004] Tuulos, V. and Tirri, H. (2004). Combining topic models and social networks for chat data mining. In Web Intelligence, 2004. WI 2004. Proceedings. IEEE/WIC/ACM International Conference on, pages $206-213$.

[Yang, 1994] Yang, Y. (1994). Expert network: effective and efficient learning from human decisions in text categorization and retrieval. In Proceedings of the 17th annual international ACM SIGIR conference on Research and development in information retrieval, SIGIR '94, pages 13-22. Springer-Verlag New York, Inc.

[Ziegler and Golbeck, 2006] Ziegler, C.-N. and Golbeck, J. (2006). Investigating interactions of trust and interest similarity. Decision Support Systems, 43(2):460 - 475. 